Article

\title{
Distance and Visual Angle of Line-of-Sight of a Small Drone
}

\author{
Kai Way $\mathrm{Li}^{1,2, *}$, Chang Sun ${ }^{1}$ and Nailiang $\mathrm{Li}^{1, *}$ \\ 1 School of Mines, China University of Mining and Technology, Xuzhou 221116, China; suncolive@163.com \\ 2 Department of Industrial Management, Chung Hua University, Hsinchu 30012, Taiwan \\ * Correspondence: kai@chu.edu.tw (K.W.L.); 0748@cumt.edu.cn (N.L.)
}

Received: 1 July 2020; Accepted: 6 August 2020; Published: 9 August 2020

\begin{abstract}
Determining the distance of the line-of-sight (LOS) of a small drone is essential in both regulating drone operation and drone operator training considering public safety. A field experiment was conducted to determine the LOS distance and visual angle of a small drone. Human participants were requested to observe a drone in one of the predetermined locations in the air. They responded whether they could see and hear the drone using a five-point scale. It was found that auditory signals were insignificant in drone detection because most of the participants could not hear the drone while they could still see the drone in most of the test locations. Logistic regression analyses were conducted to predict the probability of catching the drone visually. Two models were built considering the "definitely yes" and "definitely or probably yes" criteria of visual detection. These models may be used to estimate the LOS distance and visual angle. Assuming a 50\% probability of visual catching and the "definitely or probably yes" criterion, the distance and visual angle of the LOS for the Mavic Air drone without a protector were approximately $307 \mathrm{~m}$ and $0.065^{\circ}$, respectively.
\end{abstract}

Keywords: drone; visual line-of-sight; ground observer response; aviation safety

\section{Introduction}

Unmanned aerial vehicles (UAVs), or commonly known as drones, have been adopted for military missions for decades. With advancements of miniaturizing hardware, better affordability, and an easy-to-use design, UAVs are widely used in entertainment and applications in industry such as aerial photogrammetric survey [1], wildlife and coastal habitat monitoring [2], transportation engineering [3], parcel shipping [4], inspection of construction sites [5], disaster management [6], and movie filming [7]. Drones have even been preprogrammed to provide air shows in the night and many other entertainment purposes. Privately owned UAVs are mostly operated by an external pilot or operator on the ground using a remote-control device. The operator reads the flight information from a monitor and moves the joysticks to send a flight command [8]. UAVs with multiple propellers, especially the four-propeller ones, are widely used for commercial and personal purposes because of the performance in stability and flight control.

The emergence and popularity of the UAVs has created safety problems [9-13]. The literature indicates that the accident rate of the UAV is, in general, much higher than that of manned aircraft [14]. UAV-related ground people injuries and even fatalities have been reported $[15,16]$. In addition to safety problems, UAVs taking photos or filming videos of ground targets on private properties may also raise the concerns of privacy for the public [17]. People may feel that the UAV is taking a photo of them, which violates their privacy even if the camera on board is actually not activated. Drone invasion into a prohibited airspace has, then, become an emergent issue because of safety and privacy concerns of the public [18]. 
For the safety of the public, the aviation authorities in many countries employ different approaches to avoid drone accidents. These approaches include implementing UAV ownership requirements, drone operator training, and implementation of drone operation administration. Codes and regulations concerning these requirements have been announced. The drone registration requirement in the USA was implemented in 2015. The Federal Aviation Administration has received 300,000 registrations within only one month after announcing this requirement $[19,20]$. The drone registration requirements in China and in Taiwan were implemented in 2017 and 2018, respectively. At the end of 2018, the Civil Aviation Administration of China (CAAC) has received 287,000 drone registrations [21]. Drone operator training is achieved by pilot licensing regulations. These regulations have been implemented via a community outreach program. For example, a UAV operator license is required to operate a UAV with a takeoff weight of $7 \mathrm{~kg}$ or higher in China [22,23]. In Taiwan, a common license is required to operate a UAV of $2-15 \mathrm{~kg}$, and a professional license is required to navigate a UAV $15 \mathrm{~kg}$ or higher [24]. The drone codes, in general, prohibit a flight in a no-fly zone (NFZ) and a flight above a certain altitude in a restricted-fly zone (RFZ). A low altitude flight above crowds of people without prior approval from local authority is also not allowed [22,24,25]. Alcoholic consumption is prohibited for drone operations. In Taiwan, the alcoholic offence for pilots of civil manned aircraft also applies to drone operators [24].

The aviation authorities generally require that privately owned UAV may fly in unrestricted open space not higher than $120 \mathrm{~m}$ (or $400 \mathrm{ft}$ ) above ground level (AGL) and within the line-of-sight (LOS) during daytime $[22,25,26]$. Defining the LOS is one of the fundamental tasks in the administration of small drone operations in open spaces. The LOS may be defined using the distance between the drone and the operator and the corresponding visual angle. The visual angle is defined as "the angle a viewed object subtends at the eye" [27]. If a person watches an object with a linear size of S in a distance of $D$, the visual angle $(\theta)$ may be calculated using the following equation:

$$
\Theta=2 \tan ^{-1}(\mathrm{~S} / 2 \mathrm{D})
$$

The Civil Aeronautics Administration [24] has defined visual LOS as the far most distance between an operator and the UAV where the operator can visually catch the UAV without using cameras, telescopes, or other visual aids when observing the UAV. These visual aids do not include the ordinary lens and contact lens to correct the visual acuity of the operator. The fundamental idea of regulating drone operation within LOS is that the operators are believed to be capable of avoiding an impact of the drone with other objects whenever he or she is fully aware of the trajectory of the drone and where the drone is. The LOS is not explicitly defined in terms of either distances or visual angles in the codes of many countries such as the UK and Taiwan. The CAAC [22], however, has defined the LOS in operating a UAV as a radius of $500 \mathrm{~m}$ and an altitude of $120 \mathrm{~m}$ AGL. The distance between the drone and the operator at this altitude and radius is approximately $514 \mathrm{~m}$. Whether an operator could visually catch a drone in such a distance is questionable. It is apparent that more scientific evidence is needed to define the concept of LOS [18].

There are many factors affecting the distance of the LOS for drone navigation. The visual capability of the observer, design of the drone (size, color, and sound of motors), weather conditions (such as ratio of cloud coverage and orientation to the sun) are among the primary ones. The distance within LOS for drone operation could be quite different depending on these factors. However, how these factors affect the distance of LOS has not been reported in the literature. The study of Li et al. [28] was the first one and only one studying the LOS distance. In this study, the authors conducted a field test to determine the LOS using a Phantom 4 quadcopter. Their results indicated that the probability to catch their quadcopter $500 \mathrm{~m}$ away visually was less than $10 \%$. Assuming a $50 \%$ probability of visual capture and the "definitely or probably yes" criterion, they recommended a distance of $245 \mathrm{~m}$ as the distance of the LOS for small quadcopters. The corresponding visual angle for this LOS distance was approximately $0.065^{\circ}$.

Determination of the LOS distance of small drones is complicated. The purpose of this study is to determine the probability of visual detection of a small drone in the air in terms of drone distance. 
The LOS visual angle of this drone was also determined and discussed. In addition, the CAAC definition of the LOS distance was re-examined to determine whether this distance is appropriate for a commonly adopted small drone.

\section{Materials and Methods}

\subsection{Human Participants}

We recruited 24 healthy individuals, including 12 males and 12 females, as human participants in this study. Their ages were $22.8( \pm 2.1)$ yrs. The corrected visual acuities (in decimal) of the right and left eyes of these participants were both $1.0( \pm 0.1)$. All the participants had normal color visions and hearing functions. They were all novices without any experience in drone operation. All participants read and signed an informed consent before joining the experiment. A local ethic committee at China University of Mining Technology has approved this study (CMTU-SM-2019001).

\subsection{Unmanned Aerial Vehicle}

A Mavic Air drone (DJI ${ }^{\circledR}$, Shenzhen, China) was adopted (see Figure 1). This drone had four propellers. The weight of this drone was only $430 \mathrm{~g}$. At the ready-to-fly condition, this drone had a height and a diagonal size (propeller included) of $6.4 \mathrm{~cm}$ and $34.7 \mathrm{~cm}$, respectively. This drone might also fly with a frame surrounding the outer edge of the propeller, which is designed to protect the propellers from contacting solids such as tree leaves (see Figure 1b). The diagonal size of this drone was $38.7 \mathrm{~cm}$ when the protector was used. This Mavic Air was tested under with and without protector modes.

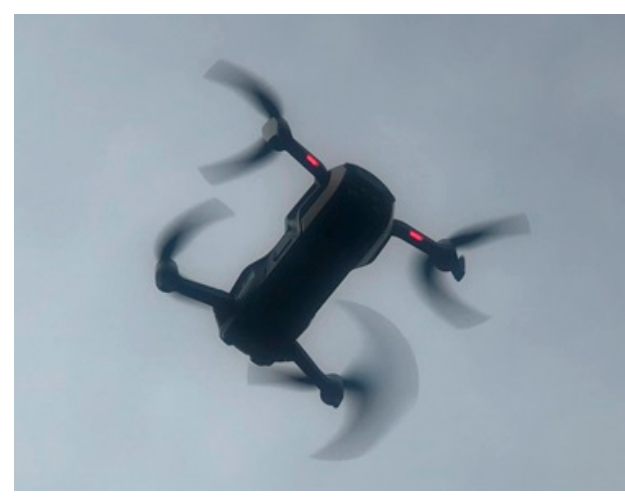

(a)

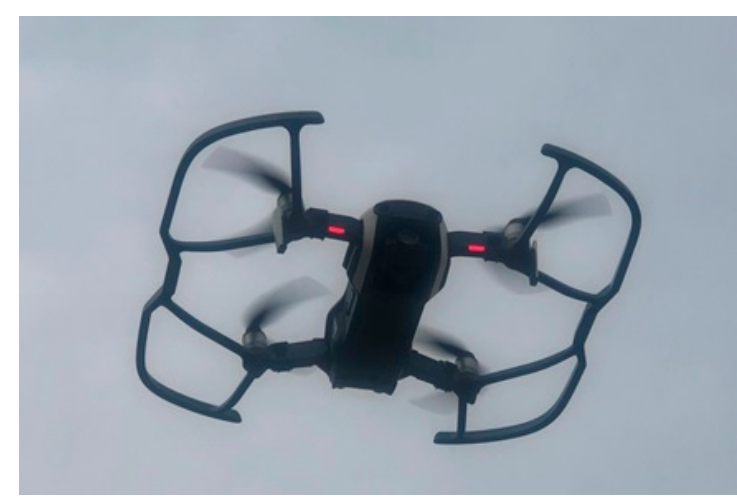

(b)

Figure 1. Mavic Air: (a) without protector and (b) with protector.

This drone encompasses both visual and satellite positioning systems. It may hover and stabilize itself in the air because of these positioning systems. The horizontal and vertical hovering accuracies were $\pm 1.5 \mathrm{~m}$ and $\pm 0.5 \mathrm{~m}$, respectively, when the satellite positioning system was used. The Mavic Air may fly when the wind was level $5(8-10.7 \mathrm{~m} / \mathrm{s})$ or less. When the wind speed was near this limit, strong wind alarm appeared on the top of the monitor warning the operator to fly carefully.

To navigate the drone, a remote controller and a smart phone are required. The remote controller has two joysticks, which allow commands of movements in eight directions (up, down, forward, backward, move left, move right, turn clockwise, and turn counterclockwise). A Mi ${ }^{\circledR} 9$ smartphone was attached to the remote controller as the monitor to display the flight information. The DJI GO ${ }^{\circledR}$ app on the smartphone was adopted as the flight control software. The location of the drone during the flight was marked as a dot on either an e-map or a satellite ground image on the monitor. In addition to this information, the primary flight information includes horizontal distance to the take-off spot, altitude, remaining power, and intensities of remote and satellite signals. The horizontal distance to 
the takeoff spot and altitude are displayed using digits on the bottom of the monitor. The intensities of remote and satellite signals and remaining power are displayed using icons on the top of the monitor.

\subsection{Test Site}

The experiment was conducted in an open space on a university campus. The test site was inside a RFZ where a flight of $60 \mathrm{~m}$ AGL was prohibited for any privately owned UAV. The participants were aligned in three rows (o in Figure 2). The take-off spot (• in Figure 2) of the drone was $3.5 \mathrm{~m}$ in front of the first row of the participants. The distance between each participant and the next one was approximately $0.5 \mathrm{~m}$. After the drone took off, it was navigated to one of the predetermined locations ( $\$$ in Figure 3) in the air $40 \mathrm{~m}$ AGL and was hovering there. The horizontal distance between the hovering position and the take-off spots was between 150 to $500 \mathrm{~m}$. Five hundred meter was taken as the farthest testing distance because none of our researchers could see the drone at this distance in a pilot trial. The distances and visual angles of the drone with and without the protector at these hovering locations are shown in Table 1.

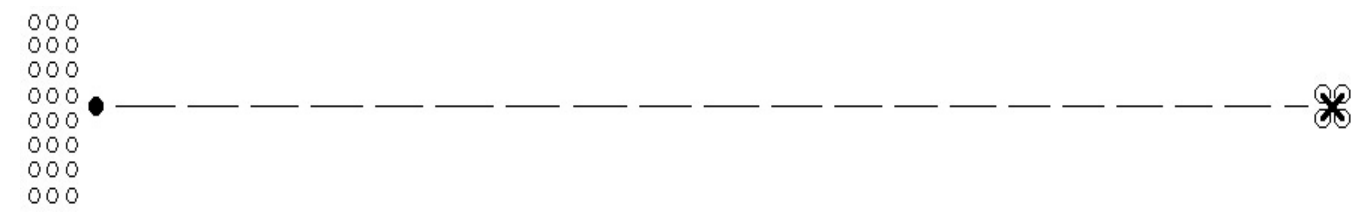

Figure 2. Locations of participants (o) and drone $\left(\circledast^{\circledR}\right)$, top view.

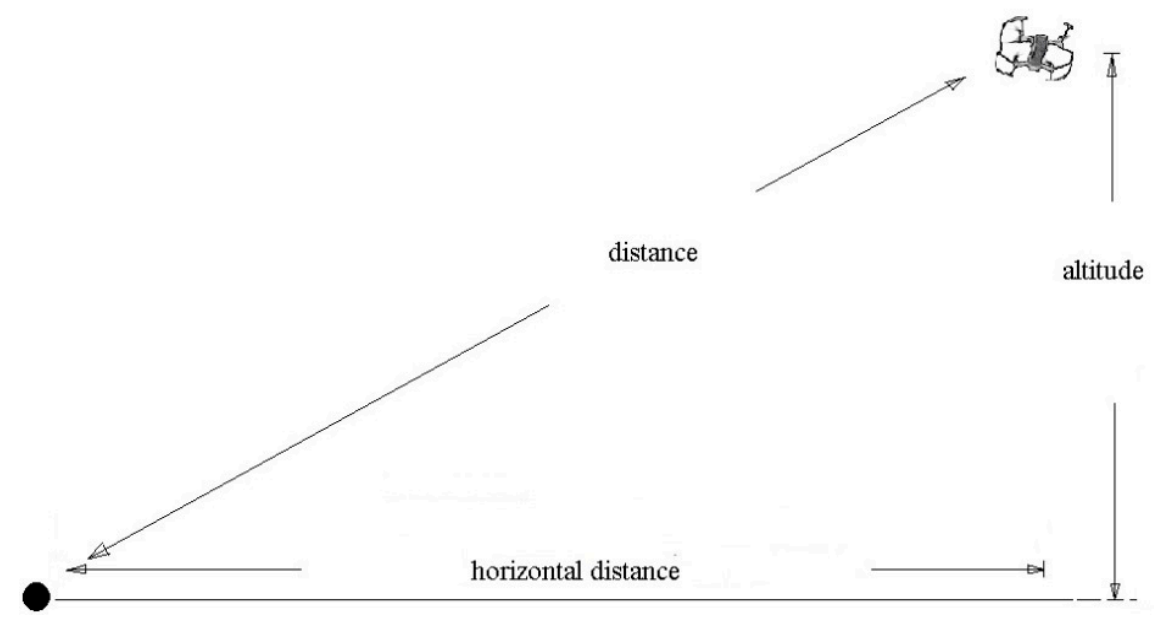

Figure 3. Distance of the drone, side view.

Table 1. Drone hovering locations and visual angles.

\begin{tabular}{cccc}
\hline & \multicolumn{3}{c}{ Visual Angle ** } \\
\hline Horizontal Distance $(\mathbf{m})$ & Distance $^{*}(\mathbf{m})$ & with Protector & without Protector \\
\hline 150 & 155 & 0.143 & 0.128 \\
200 & 204 & 0.109 & 0.097 \\
250 & 253 & 0.088 & 0.079 \\
300 & 303 & 0.073 & 0.066 \\
350 & 352 & 0.063 & 0.056 \\
400 & 402 & 0.055 & 0.049 \\
450 & 452 & 0.049 & 0.044 \\
500 & 502 & 0.044 & 0.040 \\
\hline
\end{tabular}

Note: all the altitudes were $40 \mathrm{~m} \mathrm{AGL;}{ }^{*}$ distance $=\sqrt{\text { altitude }^{2}+\text { horizontal distance }^{2}} ;{ }^{* *}$ degree, measured from the origin. 
The experiment started at 09:00 am and lasted for approximately two hours. The weather was cloudy. The wind speed on the ground, reported by the UAV Forecast ${ }^{\mathrm{TM}}$ app, was $8 \mathrm{~km} / \mathrm{h}$. This corresponds to a wind of level 2 (light breeze) in the Beaufort Wind Scale [29]. The wind direction was easterly. The relative humidity and temperature were $47 \%$ and $31^{\circ} \mathrm{C}$, respectively. The background noise and illuminance at the takeoff spot on the ground were $52 \mathrm{~dB}$ and 293 lux, respectively. They were measured using a sound level meter (Smart ${ }^{\circledR}$ Sensor, AS 824, Honk Kong, China) and a light meter (Prokit's Industries ${ }^{\circledR}$ Co. LTD, MT-4617LED-C, New Taipei City, Taiwan) before the drone was started. The visibility was $10.3 \mathrm{~km}$ [30]. The sun was on the east and $86.68^{\circ}$ to the south and $49.44^{\circ}$ above the horizon at 09:00 [31]. The participants were facing to the south west without glare in their visual field. The drone navigation path and all the hovering locations were higher than all the plants and buildings in the testing site. The background of the drone's hovering locations during the experiment, observed from the participants' area, was the cloudy sky.

\subsection{Procedure}

In the experiment, the participants stood behind the takeoff spot in the observers' area. They were facing backwards to the drone without watching the testing space when the operator was controlling the drone to fly to one of the tested locations. When the drone had reached the tested location and was floating there, the participants were requested to turn around and to observe the drone. They needed to answer the question "Have you seen the drone in the air?" on a sheet of paper. A five-point scale was adopted for the participants' responses: 1 definitely yes, 2 probably yes, 3 not sure, 4 probably no, and 5 definitely no. In addition to the visual test question, the participants also answered the question "Have you heard the sound of the drone?" using a four-point scale response: 1 heard clearly, 2 heard somewhat clearly, 3 heard indistinctly, and 4 did not hear. The participant could not communicate with any of his or her peers during the trials. They responded to the questions based on their own judgments. There was no time limit. After all the participants had completed answering the two questions, a research assistant asked them to turn around and wait for the next trial. The operator then had the drone fly to the next location.

The Mavic Air can fly no more than half an hour. When the low power signals were blinking, the operator had the drone flown back to the take-off spot and replaced the battery. The participants took a break when this occurred. The research personnel resumed the experiment after replacing the battery and guided the drone to return to the testing air space.

\subsection{Data Analyses}

The drone was flying with and without the protector. There were eight drone hovering locations (horizontal distances) for each. The drone with the protector was tested first and then the without protector mode. The order of the drone location was arranged randomly within each mode. A total of 384 ( 24 participants $\times 8$ locations $\times 2$ modes) drone detection tasks for both the visual and auditory capture were conducted. The participants' responses in catching the drone were recorded and analyzed. The pairwise $t$-test was conducted for comparison of the probabilities of drone capture between the protector modes. The significance level $(\alpha)$ was 0.05 . Logistic regression modeling was conducted to predict the probabilities of drone detection. We employed the SAS ${ }^{\circledR} 9.4$ software (SAS Institute Inc., Cary, NC, USA) for statistical analyses.

\section{Results}

Figures 4 and 5 show the probabilities of visual detection for drone flying with and without the protector, respectively. When the protector was used, the probabilities of visual detection for the "definitely yes" (DY) criterion were lower than those of the "definitely or probably yes" (DPY) criterion between the distance of $155 \mathrm{~m}$ and $452 \mathrm{~m}$. Beyond $452 \mathrm{~m}$, the probabilities of visual detection employing the two criteria coincided. The probabilities of visual catch for the DY criterion over all locations $(0.29)$ was significantly $(p<0.05)$ lower than that of the DPY criterion $(0.47)$. When there 
was no protector, the probabilities of visual catch using the DY criterion were lower than those of the DPY criterion for all the distance. The probability of visual catch for the DY criterion averaged over all locations $(0.29)$ was significantly $(p<0.001)$ lower than that of the DPY criterion $(0.44)$. The difference of the probabilities of visual catch between the two protector modes adopting the DY criterion was not significant. Neither was the difference of the probabilities of visual catch between the protector modes when the DPY criterion was adopted. We, then, focused our analysis on the visual catch data of the drone without the protector only.

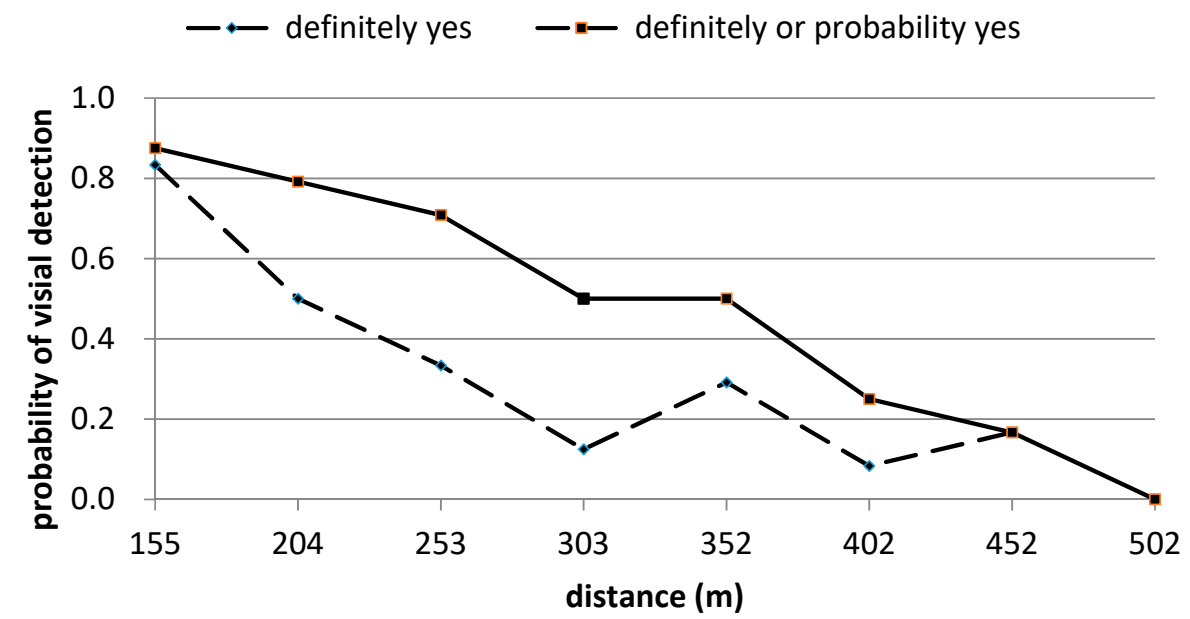

Figure 4. Probability of visual detection of the drone with the protector.

-־ definitely yes

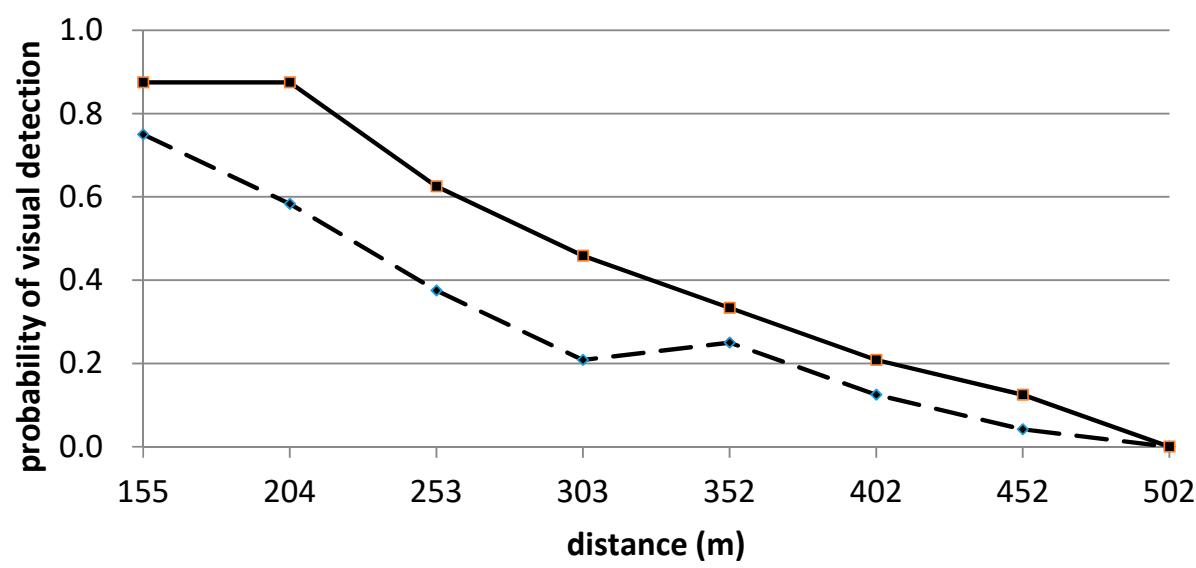

Figure 5. Probability of visual detection of the drone without the protector.

We conducted logistic regression analyses to predict the probability of visual capture versus the distance between the drone and the take-off spot. The dependent variables were $P_{1}$ and $P_{2} . P_{1}$ and $P_{2}$ are the probabilities of responding DY and DPY, respectively, for the drone hovering without the protector. The following equations were obtained:

$$
\begin{aligned}
& \operatorname{Ln}\left(\frac{P 1}{1-P 1}\right)=2.965-0.013 \times \text { distance } \\
& \operatorname{Ln}\left(\frac{P 2}{1-P 2}\right)=4.292-0.014 \times \text { distance }
\end{aligned}
$$


The coefficients of determination (or $\mathrm{R}^{2}$ ) of Equations (2) and (3) were 0.95 and 0.97, respectively. These equations may be rewritten as:

$$
\begin{aligned}
& P_{1}=\frac{e^{2.965-0.013 ? \text { diistance }}}{e^{2.965-0.013 ? \text { distance }}+1} \\
& P_{2}=\frac{e^{4.292-0.014 \text { ?distance }}}{e^{4.292-0.014 \text { ?distance }+1}}
\end{aligned}
$$

Based on Equations (4) and (5), the estimated probabilities of visual detecting the Mavic Air drone flying without the protector were obtained (see Figure 6). The ratio of drone size $(34.7 \mathrm{~cm}$ without protector) divided by the tangent of the visual angle was used to replace the distance in Equations (4) and (5). The predicted probabilities of visual capture versus the visual angle might, then, be obtained (see Figure 7).

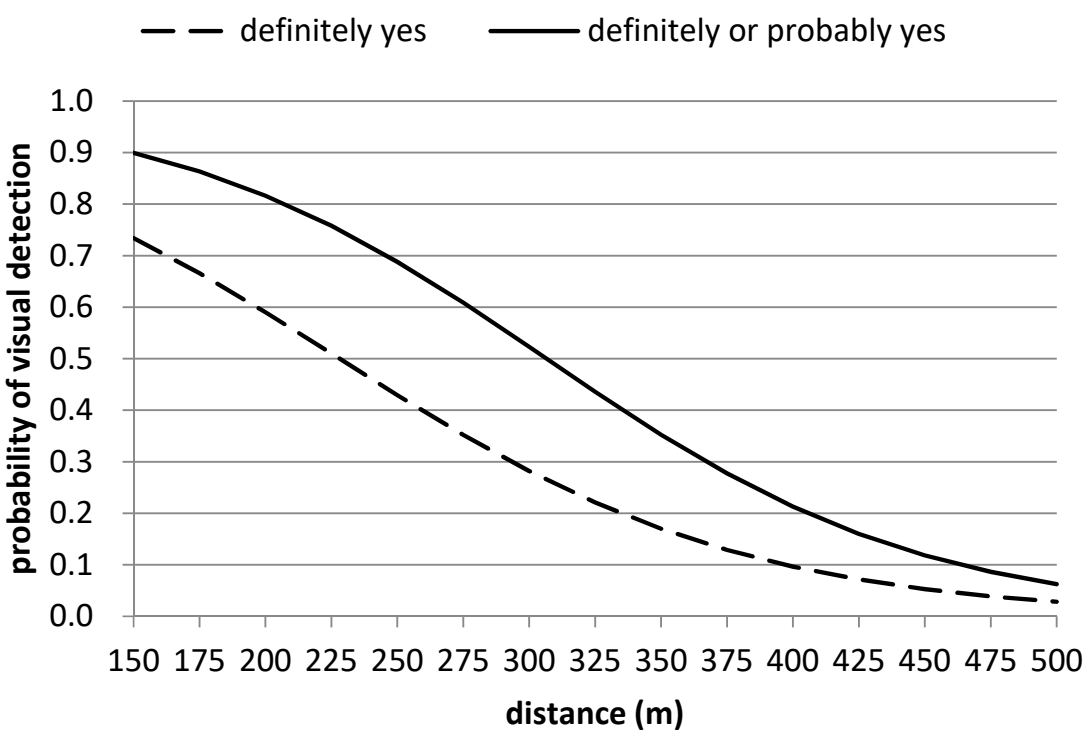

Figure 6. Estimated probability of visual detection.

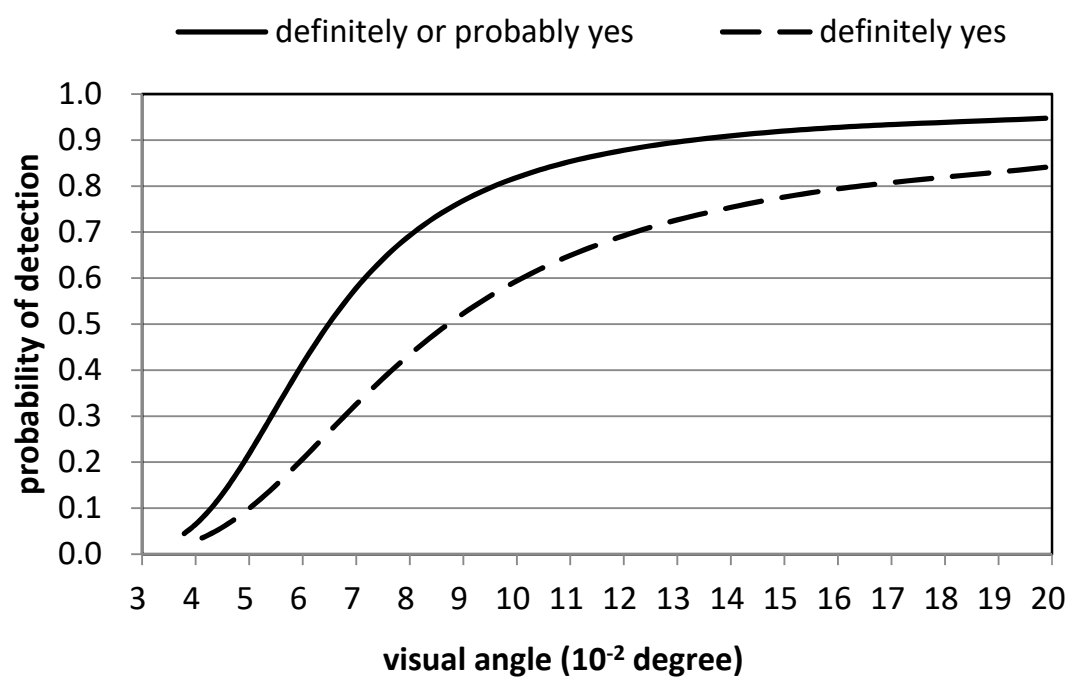

Figure 7. Probability of visual detection versus visual angle.

The predicted distances and visual angles for the LOS of the drone without the protector were calculated using Equations (4) and (5) (see Table 2). Investigating whether an operator can catch a drone in the air may be treated as a similar process of exploring the absolute threshold of target 
detection. A probability of $50 \%$ has been adopted in finding out the threshold of the intensity of a stimulus [29]. The estimated drone distances for a 50\% probability of visual catching employing the DY and DPY criteria for the Mavic Air flying without the protector, calculated using Equations (4) and (5), were $228 \mathrm{~m}$ and $307 \mathrm{~m}$, respectively. The visual angles corresponding to these drone distances were $0.087^{\circ}$ and $0.065^{\circ}$, respectively.

Table 2. Estimated distance (m) and visual angle ( $10^{-2}$ degree) for the line-of-sight (LOS).

\begin{tabular}{ccccc}
\hline & DY & \multicolumn{3}{c}{ DPY } \\
\hline Probability & Distance & Visual Angle & Distance & Visual Angle \\
\hline 0.1 & 397 & 5.0 & 464 & 4.3 \\
0.2 & 335 & 5.9 & 406 & 4.9 \\
0.3 & 293 & 6.8 & 367 & 5.4 \\
0.4 & 259 & 7.7 & 336 & 5.9 \\
0.5 & 228 & 8.7 & 307 & 6.5 \\
0.6 & 197 & 10.1 & 278 & 7.2 \\
0.7 & 163 & 12.2 & 246 & 8.1 \\
0.8 & 121 & 16.4 & 208 & 9.6 \\
0.9 & 59 & 33.7 & 150 & 13.3 \\
\hline
\end{tabular}

When the Mavic Air was flying with the protector, none of the participants could hear the sound of the drone when the horizontal distance was beyond $200 \mathrm{~m}$ at an altitude of $40 \mathrm{~m}$ AGL. When the drone was at $150 \mathrm{~m}$ away, only one participant out of $24(1 / 24)$ heard the drone indistinctly and all others could not hear at all. When this drone was flying without the protector, only two of the participants $(2 / 24)$ could hear the drone indistinctly at the horizontal distance of $150 \mathrm{~m}$ and only one participant could hear indistinctly the sound at a horizontal distance of $250 \mathrm{~m} \mathrm{(1/24)}$. None of the participants could hear when the drone was $250 \mathrm{~m}$ or farther.

\section{Discussion}

The human-machine system in operating a drone consisted of two subsystems. The first one involves accessing the flight information of the operator. The second one includes the flight control of the drone via the remote controller. Flight information may be accessed by either observing the drone directly or watching the monitor on the remote controller. When the drone is flying within a short distance, the operator could catch flying information such as the altitude, horizontal distance, and moving direction, by direct observation of the drone movements. On the other hand, when the drone is flying at a distant location with a small visual angle, direct observation of drone movements provides little information to the operator. For example, an operator may have difficulty catching a drone several hundred meters away, not to mention catching the altitude and moving direction of the drone. The operator, then, needs to rely on flight information on the monitor of the remote controller. When the operator is depending on the monitor to access the flight information, he or she may not be aware of whether the drone is within LOS unless the operator has prior LOS information in terms of distance. Providing the LOS distance is therefore important for flight control especially when the drone is at a distant location.

Even though an operator may access flight information from the monitor on the remote controller, he or she may, sometimes, need to observe the drone in the sky in order to gain feedback of the flight control. This is very important especially for novice operators. For example, a novice may not be sure whether pushing a joystick forward will lead to a forward or a backward movement because he or she may not be aware of the head orientation of the drone because quadcopters do not have a distinct head and tail in appearance. By watching the drone movement in the air, the operator may confirm whether the drone is moving in the way consistent to his or her control. This, however, can only be done when the drone is within the LOS. The LOS information will be beneficial for drone trainers to help their trainees in achieving movement compatibility in drone operation training. 
The aviation authorities, in general, require that drone operation conducted during the day and within LOS. The LOS is a spatial domain in which the operator can visually catch the drone. It may be defined [28] using the largest distance between the drone and the operator or the minimal visual angle by which the operator has a 50\% probability to catch the drone visually. Li et al. [28] found that the estimated distance and visual angle of the LOS for a DJI Phantom 4 drone were $245 \mathrm{~m}$ and $0.065^{\circ}$, respectively, when the DPY criterion was adopted. In the current study, the estimated distance and visual angle of the LOS for the Mavic Air flying without a protector were $307 \mathrm{~m}$ and $0.065^{\circ}$, respectively, considering also the DPY criterion. When a DY criterion was adopted, the estimated LOS distance and visual angle were $228 \mathrm{~m}$ and $0.087^{\circ}$, respectively. The estimate LOS distances of the Mavic Air drone in Table 2 were significantly $(p<0.05)$ higher than those in Li et al. [28]. The differences between the estimate LOS visual angles of the Mavic Air in this study and those of the Phantom 4 in Li et al. [28] were, however, insignificant.

Both LOS distance and visual angle affect how large the image is of a visual target on the retina and thus affect the likelihood of visual detection. The LOS distance is, theoretically, equivalent to the LOS visual angle. However, the effects of both the LOS distance and visual angle on the likelihood of visual detecting a drone in the air are very complicated. For example, a certain LOS distance or visual angle may be composed of different horizontal distance and altitude combinations. The sky background of the drone could be quite different for different horizontal distance and altitude combinations. Variations of the sky background will surely affect the capability of ground observers in drone detection. It was unfortunate that combinations of horizontal distance and altitude comprising of the same distance were not tested in this study. Orientation of the drone to the observer with respect to the sun also affects the visual detection. An observer may have difficulty in detecting a drone when there is glare even when the drone is within the LOS distance. Orientation of the drone and the sun also affect the reflection of the drone and thus the drone's visibility.

Larger drones are supposed to have longer LOS distances than the smaller ones. Our LOS distances of the Mavic Air were, however, significantly higher than those of the Phantom 4 in Li et al. [28]. This contradicts to what we have anticipated because the former has a smaller diagonal size $(34.7 \mathrm{~cm})$ than the latter $(59 \mathrm{~cm})$. The reason for this contradiction may be attributed to drone color and background contrast of the two studies. In Li et al. [28], the Phantom 4 drone was white and was hovering in a clear sky with hardly any cloud. The Mavic Air drone, in the current study, is a black one (observing from the bottom) and was hovering in a cloudy sky. The color contrast between a black drone in the cloudy sky and a white drone in the clear sky could be quite different. In addition, illumination could also play a role affecting the drone detecting capability of the participants. The illumination at the takeoff spot of the previous [28] and current one were 60,435 lx and $293 \mathrm{~lx}$, respectively. Such a huge difference could result in different drone detection responses. The effects of color contrast and illumination at the test site on drone detection behaviors should be explored in the future.

It is a common phenomenon that a spinning propeller looks partially transparent. This leads to the blurring of the boundary of the propeller in vision. It was one of our suspects that the actual visual angle might be smaller than that measured using the diagonal size of the propeller because the image of the two ends of the propellers might not be present in vision simultaneously. This was rejected by our results comparing the probabilities of visual detection between the drone with and without a protector. When a protector was used, there was a solid boundary outside the propellers, which clearly delineated the size of the drone. The insignificance of the difference of the probabilities of visual detection between the drone with and without the protector indicated the validity of visual angle calculation using the diagonal size of the propellers. This applies to other drone models in determining the visual angle.

Cue utilization is believed to affect the performance of visual target searching detection [32]. The observers with more visual target information, in general, have more cues and hence may have higher cue utilization than those with less target information. We could not quantify the target information of the drone hovering in the air especially when it was at a distant location. The view of a 
drone in the air is mainly composed of the spinning propellers with a drone body in the middle. When the drone is approaching the LOS location, it appears as a dot as a whole with a blurry boundary in our vision. The difference of the cue utilization of observing the Mavic Air and the Phantom 4 drones is unknown. Quantification of the cue or target visual information for drones will provide challenging research topics in the future.

The sound level generated by the Mavic Air is lower than that of a Phantom 4. Detecting a Mavic Air drone using auditory signals is more difficult than that detecting a Phantom 4 drone. At the distance of $155 \mathrm{~m}$, the probability that our participants could hear the drone indistinctly was only $8.3 \%$ when the protector was not used. This probability was even lower $(4.2 \%)$ when the protector was used. The previous study indicated that auditory signals provided partial cues in drone researching and detection. The results of the current study, however, indicated that auditory detection of the drone was insignificant in finding the LOS distance for a Mavic Air drone because none of the participants could hear the drone before it reached the visual LOS location.

The CAAC [22] has adopted the LOS distance of approximately $514 \mathrm{~m}$. Figures 4 and 5 have shown that the probability of visually catching the Mavic Air drone at a distance of $502 \mathrm{~m}$ was zero. The estimated probabilities of detecting a Mavic Air without a protector at a distance of $514 \mathrm{~m}$, based on Equations (4) and (5), were only $2.4 \%$ and $5.2 \%$ when the DY and DPY criteria were used, respectively. Our participants could not catch the Mavic Air drone at the LOS distance of the CAAC [22]. These are consistent with the findings in the literature [28].

There were limitations of this study. The first one is the atmosphere environment. It is not possible to have a controlled atmosphere environment in a field experiment. Our experiment was conducted in an open space where the sky was cloudy with light breeze and the visibility was good. These weather conditions were measured before the experiments started and some of the weather conditions, such as the gust speed, could be changed after the experiment began. Changes of the atmosphere conditions during testing could affect human responses in detecting a drone. Even the weather conditions of the current study were different than the previous study [28], the consistency between the major findings of the two studies indicate the reliability of the LOS measures of our study.

A second limitation of this study was about the participants. It was difficult to recruit experienced drone operators as human participants. The participants in this study were all novices. The literature [33-35] has pointed out that experience could have influences on the performance of visual search and detection tasks. Future research is recommended to investigate the effects of drone operating experience on the LOS distance and visual angle. In addition, our participants have normal visual acuity and color vision. Our results of visual capture could, then, be higher than those of ordinary observers who have less than normal visions.

A third limitation of our study is that the drone was navigated to the predetermined hovering location without the witness of the participants. This was because we liked to focus on the effects of distance while removing the influence of moving cues of the drone. In practice, a ground observer watches the drone flying more often than observing a stationed one. Drone movement could provide cues for drone search and detection. A moving object catches human attention and is, therefore, easier to be detected than a fixed one [36]. Our LOS distance and visual angle data might need to be revised if the participants have additional movement cues of the drone before responding. Future studies are required to incorporate the effects of drone movements on drone catching.

Finally, only one drone model was tested. Future research may be conducted to test the LOS distance and visual angle of many other models.

\section{Conclusions}

Logistic regression models were built to determine the probability of visual detection of a small drone in the air. These models allow estimation of both the LOS distance and visual angle. Assuming a probability of $50 \%$ visual catching and the DPY criterion, the distance and visual angle of the LOS for the Mavic Air drone without a protector were approximately $307 \mathrm{~m}$ and $0.065^{\circ}$, respectively. 
The LOS altitude and horizontal distance specified in CAAC [22] is not appropriate for drones like the Mavic Air because the probability of visual detecting the drone at this distance is extremely low. This paper provides valuable information for the aviation administration authorities in regulating drone operations. The findings in this paper may also be incorporated in the drone training and licensing program to help drone operators to be more capable to comply with the LOS regulations.

Author Contributions: Conceptualization, K.W.L.; methodology, K.W.L. and C.S.; formal analysis, K.W.L. and C.S.; resources, N.L.; data curation, C.S.; writing—original draft preparation, K.W.L.; writing—review and editing, K.W.L.; supervision, K.W.L.; project administration, K.W.L.; funding acquisition, N.L. All authors have read and agreed to the published version of the manuscript.

Funding: This research received no external funding.

Acknowledgments: The authors thank the support of the School of Mines at China University of Mining Technology in this study. The authors also thank the participants for their participation in the experiment.

Conflicts of Interest: The authors declare no conflict of interest.

\section{References}

1. Lin, Z. UAV for mapping-low altitude photogrammetric survey. Arch. Photogramm. Remote Sens. Spat. Inf. Sci. 2008, 31, 1183-1186.

2. Ventura, D.; Bonifazi, A.; Gravina, M.F.; Ardizzone, G.D. Unmanned Aerial Systems (UASs) for Environmental Monitoring: A Review with Applications in Coastal Habitats. Aer. Robot. Aerodyn. Control. Appl. 2017. [CrossRef]

3. Barmpounakis, E.N.; Vlahogianni, E.I.; Golias, J.C. Unmanned Aerial Aircraft Systems for transportation engineering: Current practice and future challenges. Int. J. Transp. Sci. Technol. 2016, 5, 111-122. [CrossRef]

4. Faust, A.; Palunko, I.; Cruz, P.; Fierro, R.; Tapia, L. Automated aerial suspended cargo delivery through reinforcement learning. Artif. Intell. 2017, 247, 381-398. [CrossRef]

5. de Melo, R.R.S.; Costa, D.B.; Javier, J.S.A. Applicability of unmanned aerial system (UAS) for safety inspection on construction sites. Saf. Sci. 2017, 98, 174-185. [CrossRef]

6. Erdelj, M.; Natalizio, E. UAV-Assisted Disaster Management: Applications and Open Issues, International Conference on Computing; Networking, and Communications (ICNC): Kauai, HI, USA, 2016; pp. 1-5.

7. Cleveland, P. Drama Drones: An Investigation into Integrating Drones into Real World Filmmaking in New Zealand. Master's Thesis, Auckland University of Technology, Auckland, New Zealand, 2017.

8. Vincenzi, D.A.; Terwilliger, B.A.; Ison, D.C. Unmanned aerial system (UAS) human-machine interfaces: New paradigms in command and control. Procedia Manuf. 2015, 3, 920-927. [CrossRef]

9. Waraich, Q.R.; Mazzuchi, T.A.; Sarkani, S.; Rico, D.F. Minimizing Human Factors Mishaps in Unmanned Aircraft Systems. Ergon. Des. 2013, 21, 25-32. [CrossRef]

10. Clarke, R.; Moses, L.B. The regulation of civilian drones' impacts on public safety. Comput. Law Secur. Rev. 2014, 30, 263-285. [CrossRef]

11. Loffi, J.M.; Wallace, R.J.; Jacob, J.D.; Dunlap, J.C. Seeing the Threat: Pilot Visual Detection of Small Unmanned Aircraft Systems in Visual Meteorological Conditions. Int. J. Aviat. Aeronaut. Aerosp. 2016, 3, 1-26.

12. Furfaro, D.; Celona, L.; Musumeci, N. Civilian Drone Crashes into Army Helicopter, New York Post, September 22. Available online: https:/nypost.com/2017/09/22/army-helicopter-hit-by-drone/ (accessed on 18 September 2019).

13. Clothier, R.A.; Williams, B.P.; Hayhurst, K.J. Modelling the risks remotely piloted aircraft pose to people on the ground. Saf. Sci. 2018, 101, 33-47. [CrossRef]

14. Williams, K.W. A Summary of Unmanned Aircraft Accident/Incident Data: Human Factors Implications; Technical report DOT/FAA/AM-04/24; Federal Aviation Administration, US Department of Transportation: Washington, WA, USA, 2004.

15. Forest, C. 12 Drone Disasters That Show Why The FAA Hates Drones, Techrepublic Webpage. Available online: https://www.techrepublic.com/article/12-drone-disasters-that-show-why-the-faa-hates-drones/ (accessed on 18 September 2019).

16. Democrat \& Chronicle, Domestic Drone Accidents. Democrat \& Chronicle. Available online: http://rochester. nydatabases.com/map/domestic-drone-accidents/ (accessed on 18 September 2018). 
17. Rao, B.; Gopi, G.A.; Maione, R. The societal impact of commercial drones. Technol. Soc. 2016, 45, 83-90. [CrossRef]

18. Li, K.W.; Peng, L.; Zhao, C. Human Detection of Drone Invasion in a Low Altitude Air Space: An Application of Signal Detection Theory. Hum. Factors Man. 2019, 29, 78-85. [CrossRef]

19. Federal Aviation Administration. FAA Aerospace Forecast: Fiscal Years 2016-2036. Available online: http://www.faa.gov/news/updates/?newsId=85227\&cid=TW414/ (accessed on 18 September 2019).

20. Luppicini, R.; So, A. A technoethical review of commercial drone use in the context of governance, ethics, and privacy. Technol. Soc. 2016, 46, 109-119. [CrossRef]

21. Civil Aviation Administration of China (CAAC). Civil Aviation Industry Developing Statistics. (In Chinese). Available online: http://www.caac.gov.cn/ (accessed on 18 September 2019).

22. Civil Aviation Administration of China (CAAC). Specific Unmanned Aerial Vehicle Operation Management Regulation (draft). No: AC-92-2019-01; (In Chinese). Available online: http://www.caac.gov.cn/ (accessed on 18 September 2019).

23. Civil Aviation Administration of China (CAAC). Civil Unmanned Aerial Vehicle Operator Management Regulation. No: AC-61-FS-2019-20R3; (In Chinese). Available online: http://www.caac.gov.cn/ (accessed on 18 September 2019).

24. Civil Aeronautics Administration. Civil Aviation Act, Chapter 9, Taipei, Amended 25 April 2018. 2018. Available online: https:/law.moj.gov.tw/Eng/LawClass/LawParaDeatil.aspx?Pcode=K0090001\&LCNOS= $\% 20 \% 2099-\% 209 \& L C C=2$ (accessed on 18 September 2018).

25. Federal Aviation Administration, Fly under the small UAS Rule. Available online: https://www.faa.gov/uas/ getting_started/ (accessed on 9 September 2018).

26. Civil Aviation Authority. The Drone Code. Available online: https://ronesafe.uk/wp-content/uploads/2018/ 06/Dronecode_2018-07-30.pdf (accessed on 5 September 2019).

27. Wikipedia, Visual Angle. Available online: https://en.wikipedia.org/wiki/Visual_angle (accessed on 4 August 2020).

28. Li, K.W.; Jia, H.; Peng, L.; Gang, L. Line-of-Sight in Operating a Small Unmanned Aerial Vehicle: How Far Can a Quadcopter Fly in Line-of-Sight? Appl. Erg. 2019, 81, 102898. [CrossRef] [PubMed]

29. Met Office. Beaufort Wind Force Scale. Available online: https://www.metoffice.gov.uk/guide/weather/ marine/beaufort-scale/ (accessed on 16 July 2019).

30. Bendibao. Local Weather in Xuzhou. Available online: http://xz.bendibao.com/tianqi/lishi/ (accessed on 26 June 2019).

31. Solar Calculator. Available online: http://www.yunsuan.org/app/s1904 (accessed on 19 September 2019).

32. Shekh, S.; Auton, J.C.; Wiggins, M.W. The effects of Cue Utilization and Target-Related Information on Target Detection during a Simulated Drone Search and Rescue Task. In Proceedings of the Human Factors and Ergonomics Society 2018 Annual Meeting, Philadelphia, PA, USA, 1-5 October 2018; pp. 227-231.

33. Abernethy, B. Visual search in sport and ergonomics: Its relationship to selective attention and performer expertise. Hum. Perform. 1988, 1, 205-235. [CrossRef]

34. Wen, G.; Aizenman, A.; Drew, T.; Wolfe, J.M.; Haygood, T.M.; Markey, M.K. Computational assessment of visual search strategies in volumetric medical images. J. Med. Imaging 2016, 3, 015501. [CrossRef] [PubMed]

35. Fox, S.E.; Faulkner-Jones, B.E. Eye-tracking in the study of visual expertise: Methodology and approaches in medicine. Frontline Learn. Res. 2017, 5, 29-40. [CrossRef]

36. Mcleod, P.; Driver, J.; Crisp, J. Visual search for a conjunction of movement and form is parallel. Nature 1988, 332, 154-155. [CrossRef] [PubMed]

(C) 2020 by the authors. Licensee MDPI, Basel, Switzerland. This article is an open access article distributed under the terms and conditions of the Creative Commons Attribution (CC BY) license (http://creativecommons.org/licenses/by/4.0/). 